\title{
Splicing and Coupling Losses in Hollow-Core Photonic Crystal Glass Fibers
}

\author{
J. P. Carvalho' 2, a, F. Magalhāes ${ }^{1}$, O. Frazāo ${ }^{1,2}$, J. L. Santos ${ }^{1,2}$ \\ F. M. Araújo ${ }^{1}$ and L. A. Ferreira' \\ 'INESC Porto - Institute for Systems and Computer Engineering of Poito, Portugal \\ ${ }^{2}$ Physics Department of Faculty of Science of University of Porto, Portugal \\ ajool.carvalho@inescporto.pt
}

Keywords: hollow-core photonic crystal glass fiber, coupling losses, splicing, gas sensing.

\begin{abstract}
Hollow-core photonic crystal glass fibers have a high potential for gas sensing applications, since large light-gas interaction lengths can be effectively attained. Nevertheless, in order to enhance effective diffusion of gas into the hollow-core fiber, multi-coupling gaps are often needed, which raise coupling loss issues that must be evaluated prior to the development of practical systems. In this paper, a study on the coupling losses dependence on lateral and axial gap misalignment for single-mode fiber and two different types of hollow-core photonic crystal glass fibers is carried out. In addition, an experimental technique on splicing these glass fibers is also described and some results are presented showing that low splice losses can be obtained with high reproducibility.
\end{abstract}

\section{Introduction}

Photonic crystal tibers (PCFs) [1,2] have generated a wave of excitement because of their promising properties that cannot be attained with conventional optical fibers. In these fibers, the cladding is fonned by a periodic pattern of micron-sized holes running along fiber length. The main feature of such periodic structures is that by varying the size and location of the holes, the fibers mode shape, nonlinearity, dispersion or birefringence can be tailored to reach values that are out-ofrange for conventional single mode fibers (SMF). PCFs can be divided in two main groups: solid core fibers and hollow core $(\mathrm{HC})$ fibers [3]. There are also two mechanisms for transmitting light: index-guiding, present in both solid and hollow core PCF; and bandgap guiding, which appears only in HC-PCFs.

In the hollow core PCFs light is trapped in the core not by total internal reflection, but by a photonic bandgap in the cladding that acts like an insulator for light. The HC-PCF cladding is made with hundreds of periodically spaced air holes in a silica matrix, typically arranged in a honey combed-like pattern. Because the light guiding is no longer dependent on the core's effective index, it becomes possible to create fibers that guide light in an empty or gas-filled core. Thus HC-PCFs are finding an important niche of application in the gas-sensing area since large light-gas interaction lengths becomes possible [4]. 


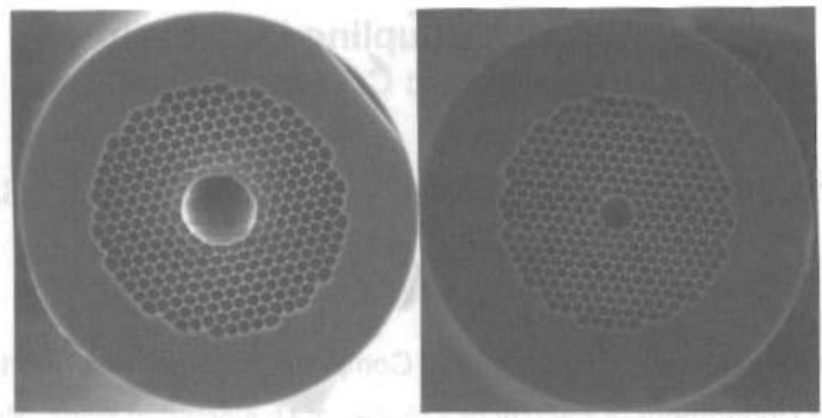

Figure I - Hollow-core photonic crystal glass fibers.

To exploit this feature it is still necessary to ensure proper diffusion of gas inside the fiber hollow-core. Several approaches can be envisaged to provide straight apertures through the cladding, creating multiple access points for the gas to reach the hollow-core. However, methods such as hole drilling often lead to asymmetrical transverse disruption of the honey combed structure resulting in intolerable losses. Multi-coupling gaps overcomes this limitation, being one of the most promising methods for the implementation of practical devices. Nevertheless, the optimum design of multiple-coupling gaps still requires a careful assessment of excess loss dependence on lateral and axial gap misalignment, particularly if it is kept in mind that the optical power in the system needs to be kept below safe levels in most hazard gas sensing applications (for example, in methane detection).

Moreover, a PCF must also be connected to standard single mode fiber to be useful in remote gas detection systems, which is still a rather delicate process. The splice losses between PCFs and SMFs have been studied in the last years by various groups [5-9]. Here a study of the splicing and coupling losses is performed for two different types of HC-PCFs: 19-cell and 7-cell HC-PCF. A brief modeling analysis and experimental measurements are presented, that allow multiple-coupling gaps loss assessment in these types of fibers.

\section{Modeling}

The coupling coefficient between two optical glass fibers can be determined from their mode profiles. If the fibers are separated by a distance $d$ (as shown in Figure 2), the field mode coupling coefficient from fiber 1 to fiber 2 is given by

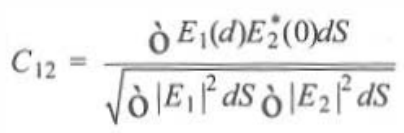

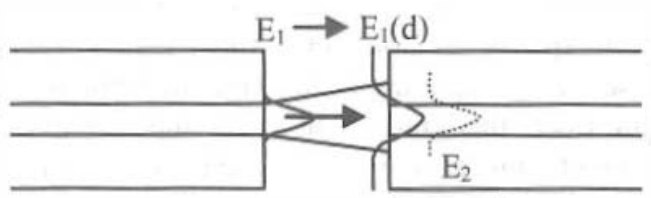

Figure 2 - Coupling between two glass fibers separated by distance $d$. 
The mode fields of a 7-cell HC-PCF and a 19-cell HC-PCF are presented in figures 3-(a) and (c), respectively. The modes of HC-PCF are not radially symmetric, therefore to simplify our analysis we radially averaged the field amplitudes of HC-PCF modes (Figures 3-(b) and 3-(d), and Figure 4). The side lobes around the central spot of the 19-cell HC-PCF are small and their amplitudes have alternating signs. Our estimation for the portion of light propagating in these side holes is $\approx 1.8 \%$. The light transmitted by these side lobes strongly diverge and will, in most cases, miss the second fiber core, particularly if some misalignment between the fibers is present. Therefore, we assume that the light in the side lobes of the 19-cell HC-PCF is effectively lost and not coupled to the other fiber.

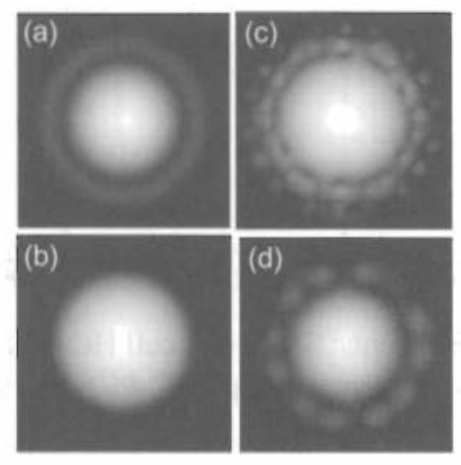

Figure 3 - (a) Mode of a 7-cell HC-PCF; (b) Radially averaged mode of a 7-cell HC PCF; (c) Mode of a 19-cell HC PCF; (d) Radially averaged mode of a 19-cell HC-PCF.

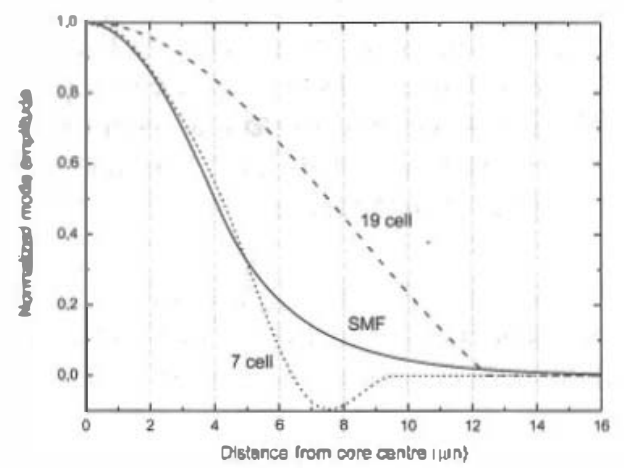

Figure 4 - Radially average of mode profiles for SMF, 7-cell HC-PCF, and 19-cell HC-PCF.

We used the mode profiles of the fibers presented in Figure 3 and simulated how they propagate in the free space between the two fibers by decomposing the beam into space Fourier components. It was found that more than $95 \%$ of the beam is in the zero-order Gaussian beam. The major difference between the fibers under analysis is then in the mode field diameter. Therefore, it is a good approximation to consider that the beams have Gaussian profiles. In this case, the optical power coupling coefficient between the two fibers in Figure 2 is given by: 


$$
\left|C_{12}\right|^{2}=\frac{4}{2+\frac{z_{2}}{z_{1}}+\frac{z_{1}}{z_{2}}+\frac{d^{2}}{z_{1} z_{2}}}
$$

where $z_{i}=p w_{i}^{2} / l$ and $w_{i}$ are the beam waists. Figure 5 shows computed results from Equation 2 relative to coupling coefficients for SMF $\rightarrow$ 7-cell HC-PCF, SMF $\rightarrow$ 19-cell HC-PCF, and 19-cell HC-PCF $\rightarrow$ 19-cell HC-PCF.

(m)

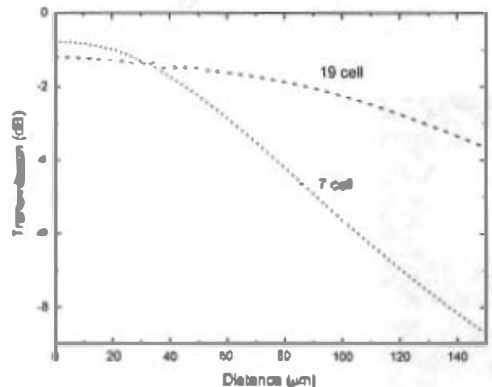

Figure 5 - (a) Coupling coefficients: SMF $\rightarrow$ 7-cell HC-PCF, SMF $\rightarrow$ 19-cell HC-PCF; (b) Coupling coefficients: I9-cell HC-PCF $\rightarrow$ 19-cell HC-PCF.

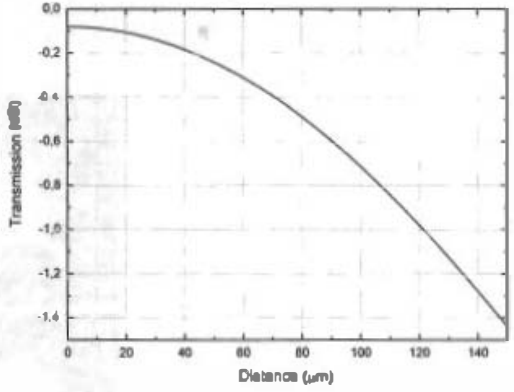

cell HC-PCF, SMF $\rightarrow$ 19-cell HC-PCF

\section{Experimental Results}

For evaluating gap coupling loss between a SMF and a HC-PCF, and also between two HC-PCFs, several measurements were made to evaluate the dependence on lateral and axial gap misalignment. A tuneable laser with $10 \mathrm{~mW}$ of maximum power was used as the optical power source. The alignment between different fibers was achieved through a system with an axial step resolution of $5 \mu \mathrm{m}$ and a horizontal/vertical step resolution of $0.1 \mu \mathrm{m}$. The light detection was made through a large area detector for the $1.55 \mu \mathrm{m}$ wavelength region.

Figure 6 shows the obtained results for axial displacement between SMF and HC-PCFs, and between two HC-PCFs of the same type. The excess loss values presented are referenced to minimal loss corresponding to direct fiber connection between the laser and the photodetector.

(a)

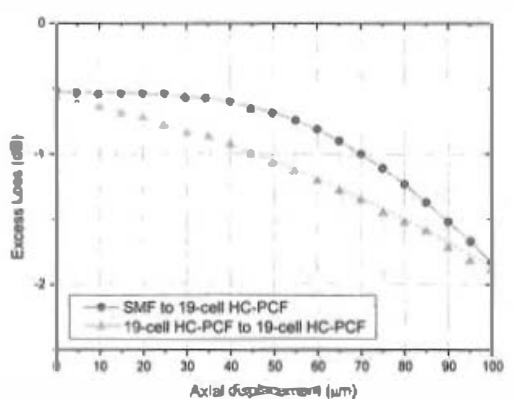
between two 19-cell HC-PCFs; (b) between a SMF and a 7-cell HC-PCF, and between two 7-cell HC-PCFs. 
The obtained results show that for similar axial displacements, the 19-cell HC-PCF has more favourable loss dependence than the 7-cell HC-PCF.

Figure 7-(a) shows the obtained results for lateral displacement between SMF and HC-PCFs. Figure 7-(b) shows the results for lateral displacement between two 19-cell HC-PCFs. During these measurements, the fibers were kept in close axial proximity.

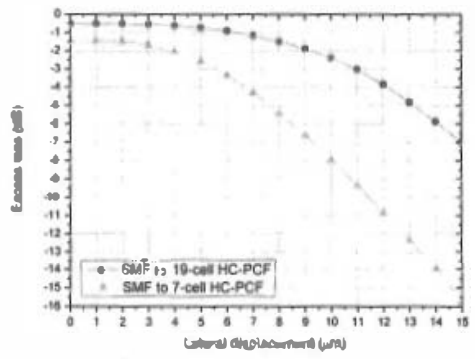

(a)

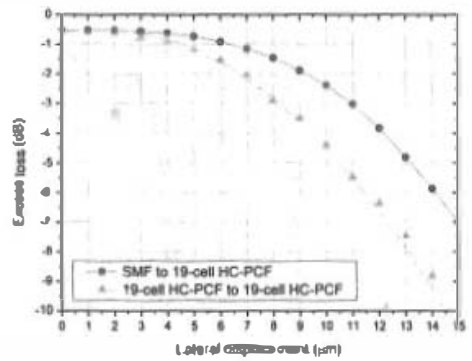

(b)

Figure 7 - Excess loss dependence on lateral displacement; (a) between a SMF and a 19-cell HC-PCF, and between a SMF and a 7-cell HC-PCF; (b) between a SMF and 19-cell HC-PCFs, and between two 19-celf HCPCFs.

These results indicate that the 19-cell HC-PCF has lower loss coupling on lateral misalignment than the 7-cell HC-PCF, considering connections with SMF and with another fiber of the same type. The coupling losses with the 7-cell HC-PCF are always larger on either lateral or axial misalignment. This behaviour was expected due to the higher mode field diameter of the 19-cell PCF when compared to both the SMF fiber and the 7-cell PCF, which are quite similar. These results indicate that 19-cell HC-PCF is less susceptible to misalignments induced during multiplecoupling gaps implementation or during system operation due to environmental effects.

Besides the evaluation of the gap induced coupling losses between these fibers, it is equally important to assess the losses associated with splicing between SMF and PCF. With the objective to reduce these losses, a splicing technique was developed based on butting the fibers and applying the electric arc discharge over the SMF region, in a process illustrated in Figure 8. During the splicing process the results for the losses were obtained using the well known cutback technique, by injecting light into the SMF-28 ${ }^{\mathrm{TM}}$ from a tuneable laser source operating at $1550 \mathrm{~nm}$. The basic splicing procedure was described elsewhere [9], but was now optimized for these fiber types. First, the fibers were automatically joined to a distance of $25 \mu \mathrm{m}$ of each other (the region where the arc usually discharge) as seen in Figure 8-a). After this stage, the fusion process proceeded manually. The hollow core photonic glass fiber position was kept fixed and then the SMF- $28^{\mathrm{TM}}$ was moved against the HC-PCF fiber, using the translation stage of the machine. The final step was to perform the electric arc discharge.
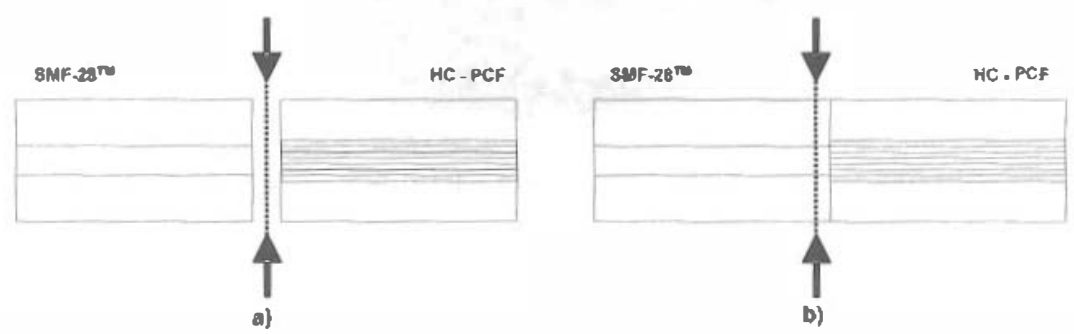

Figure 8 - Splicing technique between SMF and HC-PCF fibers. a) Result of splice machine automatic jointing of the fibers (the $25 \mu \mathrm{m}$ gap between both fibeis is seen); b) the HC-PCF is fixed, the SMF-28 ${ }^{\text {TM }}$ is moved on up to fiber contact and then il is periormed the electric arc discharge. 
To get as much information as possible about the performance achievable with this splicing technique, spectral results were obtained instead of the more straightforward splicing loss figure. These results are presented in Figure 9. From them it was concluded that the better electric arc splicing parameters in what concerns loss minimization and reproducibility are: arc current of $13.535 \mathrm{~mA}$ and a time discharge of $300 \mathrm{~ms}$.

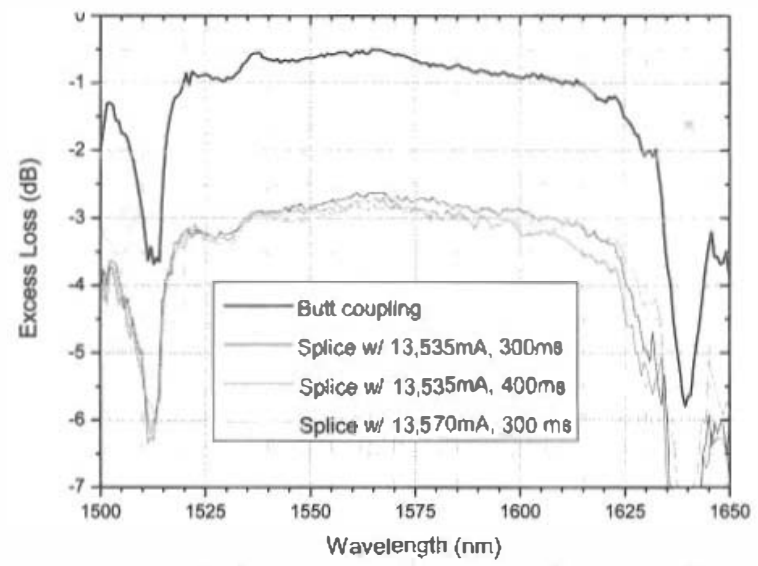

Figure 9 - Experimental results showing the loss spectral dependence relative to splicing between SMF and 19cell HC.PCF

Figure 10 shows photographs of splices obtained under different conditions. The physical shapes of the different splices do not significantly affect the splice losses between SMF and HC-PCFs. Nevertheless, even using optimum splice parameters the coupling efficiency is always lower when compared with straight butt-coupling.

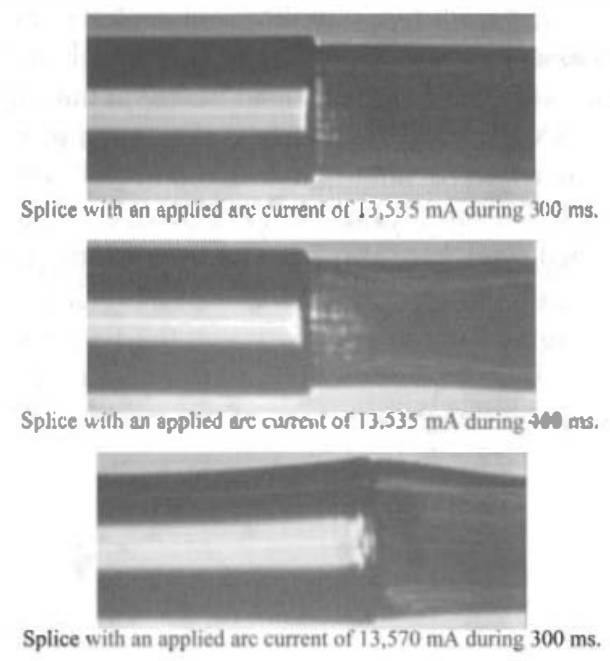

Figure 10 - Photographs of the different splices between SMF and 19-cell HC-PCF with loss results showed in Fig. 9. 


\section{Conclusion}

In this work, an analysis of the coupling losses associated with axial and lateral displacement between SMF and HC-PCF glass fibers was performed. It was found that coupling involving 19-cell HC-PCF presents lower losses when compared with those relative to the 7-cell HC-PCF. Moreover, in all cases the obtained results are encouraging towards the implementation of practical multiplecoupling gap based gas sensing systems.

It was also studied the process associated with the fusion splicing of SMF-28 $8^{\mathrm{TM}} / \mathrm{HC}$-PCF glass fibers. The study carried out indicates that it is feasible to have reproducible splice losses between 19-cell HC-PCF and SMF of $\sim 2 \mathrm{~dB}$.

\section{Acknowledgements}

This work was partially developed within the European Project NextGenPCF, supported by IST in the 6th Framework R\&D Programme. The authors acknowledge J. C. Knight and R. AmezcuaCorrea from Department of Physics, University of Bath, for providing the hollow-core photonic crystal fibers used in the experiments.

\section{References}

[1] J. C. Knight, "Photonic crystal fibres", Nature, Vol. 424, No. 14 August, pp. 847-851 (2003).

[2] P. Russel, "Photonic Crystal Fibres", Science, Vol. 299, No. 17 January, pp. 358-362 (2003).

[3] C. M. Smith, N. Venkataraman, M. T. Gallagher, D. Müller, J. A. West, N. F. Borrelli, D. C. Allan,K. W. Koch, "Low-loss hollow-core silica/air photonic bandgap fibre", Nature, No. 7 August, Vol. 424, pp. 657-659 (2003).

[4] Y. L. Hoo, W. Jin, C. H. L. Ho, D. N. Wang, S. C. Ruan, "Design and modelling of a photonic crystal fiber gas sensor", Applied Optics 42, 3509 -3515 (2003).

[5] J. H. Chong, M. K. Rao, "Development of a system for laser splicing photonic crystal fiber", Optics Express 11, 1365-1370 (2003).

[6] B. Bourliaguet, C. Paré, F. Ėmond, A. Croteau, A. Proulx, R. Vallée, "Microstructured fiber splicing", Optics Express 11, 3412-3417 (2003).

[7] D. L. Bisbee, "Splicing silica fibres with an electric arc", Applied Optics 15, 796 -798 (1976).

[8] J. T. Lizier, G. E. Town, "Splice losses in holey optical fibres", IEEE Photonics Technology Letters 13, 794-796 (2001).

[9] O. Frazão, J. P. Carvalho, H. M. Salgado, "Low loss splice in a microstructured fibre using a conventional fusion splicing", Microwave and Optical Technology Letters, Vol. 46, No. 2, July 20, 172-174 (2005). 